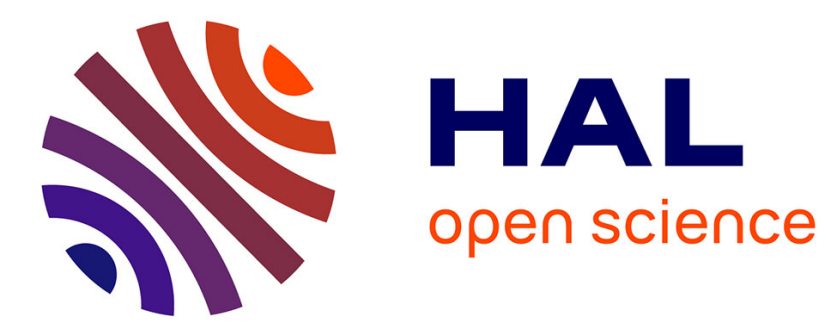

\title{
Application des techniques écologiques aux reboisements en Tunisie
}

Jean Timbal

\section{To cite this version:}

Jean Timbal. Application des techniques écologiques aux reboisements en Tunisie. Revue forestière française, 1971, 23 (1), pp.25-37. 10.4267/2042/20472 . hal-03395058

\section{HAL Id: hal-03395058 \\ https://hal.science/hal-03395058}

Submitted on 22 Oct 2021

HAL is a multi-disciplinary open access archive for the deposit and dissemination of scientific research documents, whether they are published or not. The documents may come from teaching and research institutions in France or abroad, or from public or private research centers.
L'archive ouverte pluridisciplinaire HAL, est destinée au dépôt et à la diffusion de documents scientifiques de niveau recherche, publiés ou non, émanant des établissements d'enseignement et de recherche français ou étrangers, des laboratoires publics ou privés. 


\section{APPLICATION DES TECHNIQUES ÉCOLOGIQUES AUX REBOISEMENTS EN TUNISIE}

Class. Oxford $232: 181(611)$

J. TIMBAL

Depuis son indépendance, la Tunisie, consciente du capital représenté par son patrimoine forestier, a entrepris, avec l'aide de la coopération bilatérale et internationale, de le restaurer et de le mettre en valeur.

C'est ainsi que dans ses plans quadriennaux, elle s'est donnée en particulier pour buts l'aménagement de la totalité de ses forêts naturelles et le reboisement de très vastes surfaces.

Outre son rôle de défense des sols contre l'érosion, le reboisement a pour objectif la fourniture, à plus ou moins long terme, de matière première lígneuse dont la Tunisie est pour l'instant, cruellement déficitaire.

Chaque année, en automne, une fête officielle, la «Fête de l'arbre ", a pour ambition de sensibiliser l'opinion publique à ce problème et de lui inculquer le respect de l'arbre, à la fois ressource et protection contre l'érosion. En faisant réaliser des reboisements populaires et collectifs, elle entend montrer que ce doit être l'affaire de tous.

Cet effort de reboisement et d'aménagement a d'abord et surtout porté sur la partie Nord du pays, là où l'importance des précipitations devait rendre, a priori, ces investissements les plus productifs.

L'importance que revêt la connaissance des divers milieux naturels et des facteurs écologiques qui les déterminent, est bien connu à tous les stades de la mise en valeur: du stade des aménagements forestiers, pour distinguer les zones à vocation vraiment forestière des autres, au stade du reboisement des zones dégradées pour le choix de l'essence à utiliser et des secteurs où cette dernière pourra être introduite avec succès.

Nous voulons présenter succinctement la méthode originale qui a été mise au point en Tunisie et que nous avons appliquée sur une vaste échelle à l'étude de tous les périmètres de reboisement du Nord de ce pays durant les années 1969 et 1970. Cette méthode est le fruit des résultats acquis durant ces dernières années par les diverses sections de l'Institut de Reboisement de Tunis et une de nos tâches a été de former des agents tunisiens à son utilisation. 
Avant de poursuivre, il nous paraît nécessaire de définir rapidement les particularités physiques, climatiques et biologiques de cette zone, qui comprend essentiellement ce que l'on a coutume d'appeler la Kroumirie et les Mogods. Elle est limitée :

- Au Nord, par le rivage méditerranéen.

- Au Sud, par la vallée de la Medjerdah.

- A l'Ouest, par la frontière algérienne.

- A l'Est, par la plaine de Bizerte-Mateur.

Selon Emberger, cette région est située dans les étages bioclimatiques humide et subhumide, ce qui signifie, en particulier, que la pluviosité y varie de 500 à $1.200 \mathrm{~mm}$ en moyenne. Ce sont les plus fortes de Tunisie et elles sont concentrées surtout sur la période hivernale. La considération des minima des mois les plus froids permet en outre de distinguer plusieurs variantes:

- une variante à hiver chaud qui est étroitement localisée le long du littoral méditerranéen et qui se caractérise par la présence du Chêne Kermes (Quercus coccifera),

- une variante à hiver doux,

- une variante tempérée très générale sur les hauteurs,

- une variante fraîche exceptionnelle et localisée en quelques rares points élevés.

Topographiquement, c'est une région montagneuse mais où l'altitude n'est jamais très élevée (1.010 $\mathrm{m}$ au Dj. Bir, point culminant de la région), et où les formes du relief ne sont pas très accusées. Ceci à cause de l'érosion et de la nature du substratum géologique (Flysch oligocène acide).

Sur le plan de la végétation, c'est le domaine de la suberaie et, dans les parties les plus basses, de ce que l'on appelle traditionnellement l'O/eo-Lentiscetum (groupement à Oléastre et Pistachier Lentisque). Les parties les plus élevées et la plupart des thalwegs sont occupés par des peuplements de Chêne Zeen (Quercus faginea var. murbeckii). A elles seules, les forêts de Chêne-liège couvrent environ 100.000 ha, pratiquement d'un seul tenant. En effet, si on excepte quelques crêtes non boisées battues par les vents et les zones de culture et de parcours, l'ensemble de ces régions possède une couverture végétale, plus ou moins dense certes, mais continue.

En Tunisie, l'Etat a le monopole de la propriété forestière. II en résulte que, dans les Mogods, et plus encore en Kroumirie, la quasi-totalité du territoire lui appartient. L'administration forestière qui le représente, joue donc un rôle essentiel dans la vie sociale et économique de ces régions. En particulier, du fait de la quasi-inexistence du secteur industriel et du faible développement du secteur agricole, elle constitue pratiquement la seule possibilité d'embauche pour les habitants. C'est là une situation assez exceptionnelle pour être soulignée.

Une autre conséquence de cet état des choses est que, dans une contrée où la surface agricole utile est faible et limitée, l'expansion démographique provoque une pression très forte de la population autochtone sur le domaine forestier. Cela se traduit par une charge en bétail croissante ou même par des occupations humaines et des défrichements illicites. Ceci ne va pas sans poser de nombreux et délicats problèmes aux gestionnaires et aux responsables des aménagements.

II ne peut en effet y avoir d'aménagement forestier pleinement satisfaisant tant que ce problème social n'aura pas été résolu. De par sa nature même il est politique. II 
n'est guère possible qu'un aménagement forestier, puisqu'il concerne la presque totalité des surfaces d'une région, ne soit pas le reflet des préoccupations de politique sociale du gouvernement. Cette dernière consiste actuellement à regrouper dans des villages neufs, les habitants dispersés dans des " douars " ou dans des "gourbits " isolés au sein de la forêt.

Faute de ressources extérieures et de terres arables en quantités suffisantes, cela ne supprimera pas totalement la pression sur la forêt et surtout le surpâturage. II paraît difficile de ne pas avoir à faire "la part du feu ", c'est-à-dire de rétrocéder aux communautés villageoises ainsi constituées une partie du territoire (celle dont la vocation forestière s'avère faible ou nulle par exemple) et de "cantonner " les droits d'usage, comme cela a été fait en France à partir du XVIIIE siècle.

Cette contrainte sociale retentit également sur les reboisements. En fait, la création de chantiers nationaux de reboisement dans cette région, a d'abord eu pour but de fournir du travail aux habitants (d'où le nom ancien de " chantiers d'assistance »).

A l'heure actuelle oủ le souci d'efficacité technique et de rentabilité prime de plus en plus, le choix de l'emplacement de ces chantiers résulte encore souvent, en grande partie, de considérations sociales et politiques locales.

Etant donné le petit nombre d'années que s'est fixé comme délai le Plan Tunisien, l'aménagement, d'emblée, de l'ensemble des forêts naturelles selon des méthodes sûres et éprouvées, était impossible. Pour le secteur de la suberaie de la Kroumirie et des Mogods qui nous intéresse ici, on s'est contenté d'aménager les peuplement ligneux en bon état en laissant de côté, pour l'instant, les zones trop dégradées ou asylvatiques.

Celles-ci forment soit, purement et simplement, des zones dites " hors aménagement », soit, à l'intérieur d'une série aménagée, un quartier dit de " transformation ". Nous reviendrons sur ce dernier point un peu plus loin.

Avec l'achèvement et la publication des premiers procès-verbaux d'aménagement, d'importantes surfaces ont ainsi été affectées au reboisement, l'aménagiste prèvoyant même le calendrier des opérations. Ce dernier s'étale généralement sur toute la durée de validité de l'aménagement, soit 25 ans en moyenne.

Cela doit être un premier pas dans la rationalisation des chantiers de reboisement. Leur emplacement ne sera plus désormais fixé au hasard ou selon les desiderata des autorités locales, mais intégré au plan d'aménagement de la zone considérée.

\section{LES PREMIERES REALISATIONS DE REBOISEMENT}

Déjà, sous le protectorat français, quelques plantations avaient été réalisées, principalement à base d'Eucalyptus (E. camaldulensis, E. gomphocephala, E. maideni etc...). C'est surtout dans les premières années qui suivirent l'accession à l'indépendance que cette politique fut développée.

Mais les résultats ne furent pas toujours ceux escomptés ; une forte mortalité fut constatée, souvent même après plusieurs années d'apparente réussite. Elle est due à des causes diverses parmi lesquelles la pullulation d'insectes parasites, qui profitent de la moindre résistance des essences mal adaptées aux conditions de milieu quil leur était offertes (1).

L'inadaptation écologique explique les ẻchecs similaires constatés avec des résineux comme le Pin de Monterey dont la sensibilité aux parasites s'est révélée très grande. 


\section{CHOIX DES ESSENCES DE REBOISEMENT}

Ce choix découle actuellement des résultats obtenus par la Recherche Forestière Tunisienne en matière d'introduction d'essences exotiques et de possibilité d'emploi des essences autochtones. Ils ont été acquis :

- d'une part, grâce à l'implantation d'un vaste réseau d'arboretums où furent introduites de nombreuses essences exotiques (Eucalyptus et Pins essentiellement). Ces arboretums ont été créés à partir de 1957 par M. A. Schoenenberger dans le cadre de la Station de Recherches Forestières de Tunis. Ils ont été complétés depuis 1965 par l'Institut National de Recherches Forestières Tunisiennes, assisté par le projet "Institut de Reboisement " du Fonds Spécial des Nations Unies, dirigé par M. J. Marion ;

- d'autre part, grâce à la mise en évidence et à l'étude, dans les plus vieilles plantations, des corrélations pouvant exister entre leur degré de réussite (ou d'échec), et les facteurs du milieu les plus facilement accessibles (essentiellement le sol et la végétation). Cette méthode s'est révélée particulièrement riche en enseignements ;

- enfin, par l'étude, dans les forêts naturelles, des interrelations entre la productivité ligneuse, le sol et la végétation.

Une des premières conclusions de l'Institut de Reboisement de Tunis fût de préconiser une réduction très considérable de l'emploi des Eucalyptus dans les reboisements, au profit des résineux locaux.

Des considérations économiques à long terme font que le nombre de ces essences est faible. Ce seront désormais :

\section{- Le Pin pignon (Pinus pinea L.).}

Introduite de très longue date en Afrique du Nord, cette essence se montre particulièrement plastique (tolérante en particulier envers la teneur en calcaire et la "lourdeur" des sols). Outre son intérêt comme producteur de bois, le Pin pignon a un intérêt " fruitier " qui est loin d'être négligeable et qui peut même devenir prépondérant là où la qualité intrinsèque du sol interdit l'espoir de rendements ligneux satisfaisants.

\section{- Le Pin maritime (Pinus pinaster Ait. $=$ P. maritima Mill.).}

Le nom exact de l'espèce de Pin que l'on trouve dans la région de Tabarka est très controversé. C'est en 1883 que l'exploration botanique dirigée par E. Cosson en Tunisie septentrionale découvrit, dans la région de Tabarka des peuplements de Pins qu'elle rattacha à l'espèce Pinus pinaster Soland. En 1947, Huguet del Villar reprenant la question du Pin maritime nord-africain, fait de la race algéro-tunisienne, une variété : P. pinaster Soland. var. Renouii H. del Villar. En 1953, Mlle Y. de Ferre du Laboratoire Forestier de Toulouse, reprend le problème de la répartition respective du Pin maritime et du Pin mésogéen. Critiquant la validité des critères anatomiques et morphologiques distinctifs donnés par $\mathrm{H}$. del Villar, elle refute l'existence de sous-espèce ou de variété distincte de P. pinaster en Afrique du Nord. Elle rattache les peuplements existant à l'espèce Pinus mesogeensis Fieschi et Gaussen. C'est pratiquement l'opinion adoptée par Debazac (1964), dans son manuel des conifères.

D'un point de vue pratique cette polémique a peu d'intérêt. II reste que le binome Pinus pinaster Soland. var. Renouii $\mathrm{H}$. del Villar, même s'il est incorrect taxonomiquement, a l'avantage de désigner sans ambiguitté cette réalité biologique qu'est la race de $P$ in du littoral algéro-tunisien, la race de Tabarka, dont les caractéristiques génétiques sont bien particulières et dont l'amélioration a été commencée. Dans cette région il forme des peuplements plus ou moins en mosaïque avec les faciès thermophiles de la suberaie (faciès à Teucrium fruticans). 
Belle réussite du Pin maritime sur les landes à Fougère aigle du Djébel Ez-Zouza (Amdoun 20 série)

Photo TIMBAL

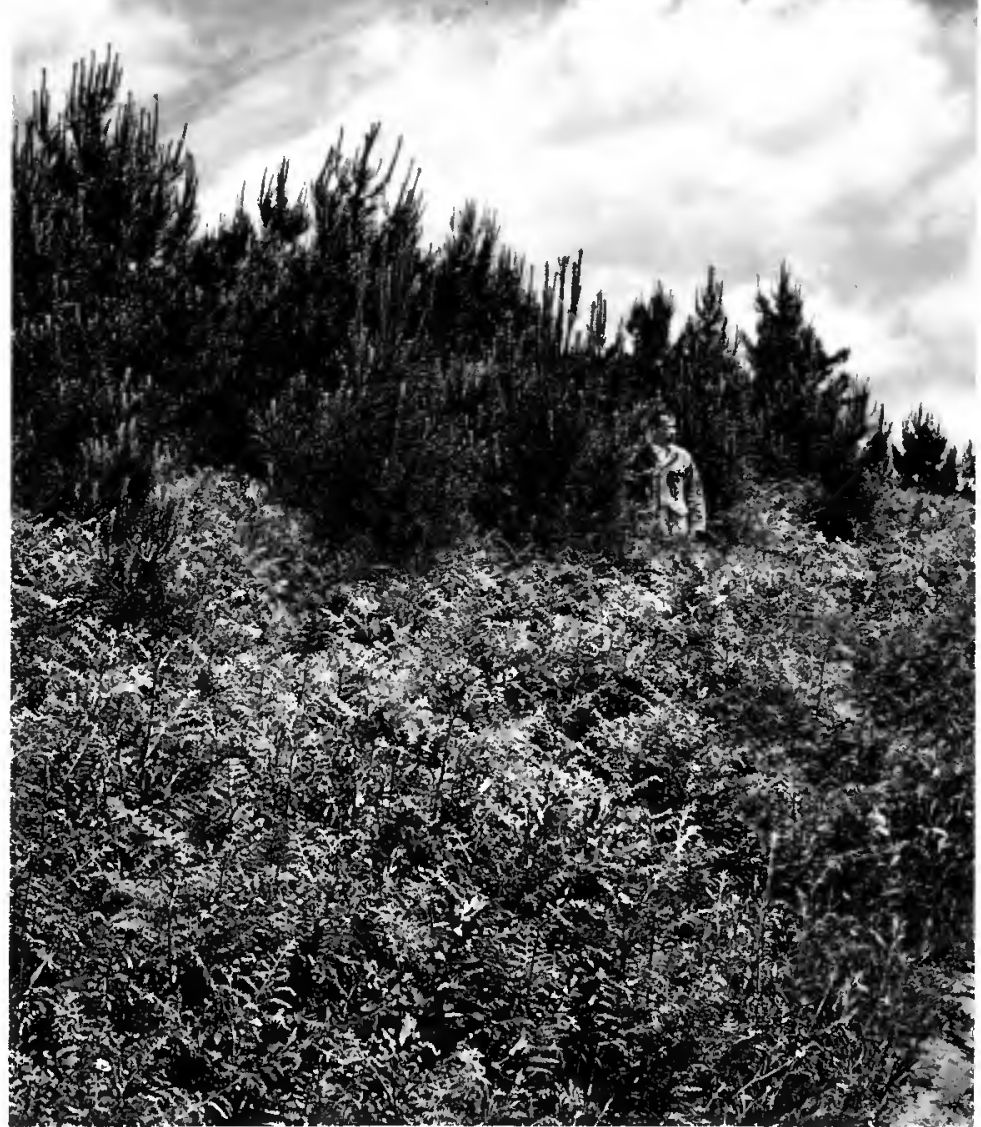

\section{- Le Pin d'Alep (Pinus halepensis Mill.).}

Il est indigène dans tout le Nord de la Tunisie. Quoique son domaine soit essentiellement constitué par les zones calcaires qui s'étendent au Sud de la vallée de la Medjerdah (Dorsale tunisienne), on le retrouve disséminé au sein de la suberaie, soit sous forme de petits peuplements localisés sur des îlots calcaires, soit sous forme d'individus isolés.

\section{— Le Cyprès toujours vert (Cupressus sempervirens L.)}

Son indigénat est supposé dans quelques localités de la dorsale tunisienne (Maktar, Ousseltia...). C'est une essence calcicole très rustique.

\section{- Le Pin de Monterey (Pinus radiata D. Don = P. insignis Dougl.).}

II pourra être utilisé de façon très prudente sur les sols les plus riches (les plus humifères et les plus profonds).

- Les Eucalyptus pourront continuer à être utilisés dans de très faibles proportions et uniquement sur les sols les plus riches et les mieux alimentés en eau (terrasses alluviales, thalwegs, etc...). 
On peut faire deux séries de remarques à propos de cette liste.

Après la première période de reboisement en Eucalyptus, qui s'est soldée par un échec relatif, ce choix marque un retrait, un repli vers des essences indigènes écologiquement mieux adaptées et dont les performances de croissance, si elles sont peutêtre au départ moins spectaculaires, sont, en fin de compte, plus sûres.

Cette liste a un caractère provisoire. Dans quelques années, et plus sûrement encore dans quelques dizaines d'années, les résultats des introductions en arboretums et ceux des essais de comparaison de provenances, indiqueront avec plus de précision les essences les plus intéressantes, pour leur production ligneuse et leur adaptation aux conditions écologiques locales.

\section{L'ETUDE DES MILIEUX}

Un des principaux résultats acquis par la Recherche Forestière en Tunisie, a été de démontrer la très grande hétérogénéité, surtout édaphique, de ce secteur Nord-Ouest, qui passait jusqu'alors pour homogène.

En effet, dans toute la Kroumirie et les Mogods, le substratum géologique est constitué à plus de $90 \%$ par un flysch oligocène acide que l'on a confondu longtemps avec l'un de ses faciès: le grès de Numidie.

II y a en réalité trois faciès principaux :

- un faciès gréseux. C'est le grès de Numidie, acide, qui constitue l'armature de tous les reliefs, mais qui affleure rarement sur des surfaces qui ne soient pas négligeables ;

Maquis de degradation de la suberaie dans la 3e serie d'Oued-Zeen (maquis à Erica scoparia, Lavandula stoechas et Halimium halimifollum), destinè à être reboisé en Pin maritime 
- un faciès argileux (argiles bariolées), très important en surface ( $80 \%$ environ). II se rencontre sur tous les versants ;

- un faciès marneux assez répandu, surtout à la périphérie du massif kroumirien. II constitue les zones les plus érodables, principalement les parties basses des pentes.

Le colluvionnement qui affecte toutes les pentes complique évidemment ce schéma.

Les autres substrats géologiques que l'on peut rencontrer dans cette région sont

- soit des calcaires crétacés situés sous la forme de rares pointements rocheux, à l'intérieur, ou, plus souvent, à la périphérie de la zone du flysch oligocène;

- soit du Trias diapirique pouvant se présenter lui aussi sous des faciès aussi différents que du gypse pur, des cargneules ou des brèches.

Cette hétérogénéité des substrats entraîne une hétérogénéité des sols et donc de leur aptitude au reboisement.

- Sur les grès, ou sur les colluvions gréseuses, se sont constitués des sols bruns lessivés ou des sols lessivés filtrants, relativement bien structurés. S'ils ne sont pas trop érodés, ils conviennent bien au Pin maritime.

- Sur les argiles se sont également constitués des sols allant des sols bruns acides aux sols lessivés et aux sols podzoliques. Mais ils présentent tous dans leurs horizons inférieurs des phénomènes d'hydromorphie plus ou moins marqués (pseudogley) constituant un facteur limitant pour le reboisement. Suivant la profondeur à laquelle on rencontre le pseudogley, c'est-à-dire, en pratique suivant l'état d'érosion du sol, son aptitude sera variable : si l'hydromorphie reste localisée en profondeur, le Pin maritime pourra encore convenir pourvu que la structure et la texture ne soient pas trop défavorables; si l'hydromorphie se manifeste à faible profondeur, seul le Pin pignon pourra résister ; enfin, si l'hydromorphie est superficielle (sol érodé ou sol hydromorphe), l'aptitude au reboisement paraît nulle dans l'état actuel de nos connaissances. Mieux vaut alors y essayer des cultures fourragères comme celle d'une graminée indigène : Oryzopsis miliacea var. thomasii.

- Sur les marnes, très érodables, on a des sols peu évolués souvent à caractère vertique, inaptes au reboisement, mais dont la potentialité fourragère est très élevée. On peut y cultiver soit des écotypes améliorés, soit des races sauvages de "Sulla " (Hedysarum coronarium) ou d'Alpiste (Phalaris canariense).

- Les calcaires portent généralement des sols bruns calcaires plus ou moins érodés, convenant bien au Pin d'Alep ou au Cyprès toujours vert.

- Les zones triasiques ont des sols qui varient suivant les faciès. Les sols bruns acides développés sur des colluvions de brèches sont toujours d'excellents sols, bien drainés et bien structurés. Le Pin maritime y donne de très bons résultats. lis portent une végétation naturelle dominée par la Fougère aigle (Pteridium aquilinum) et la Ronce (Rubus ulmifolius).

L'étude d'un grand nombre de périmètres de reboisement a montré qu'en Kroumirie $40 \%$ seulement, en moyenne, des surfaces étudiées étaient aptes à être reboisées.

Ce pourcentage est inférieur dans les Mogods du fait de la dégradation des sols.

II est donc indispensable en Tunisie, comme ailleurs, pour réussir les reboisements prévus par les plans quadriennaux, de faire l'étude écologique préalable des zones concernées, c'est-à-dire des zones dont l'aménagiste a prévu la transformation.

La conception de la transformation s'est d'ailleurs sensiblement modifiée et précisée.

Dans les premiers aménagements ce terme signifiait le remplacement d'un peuple- 


\section{CARTE DES MILIEUX}

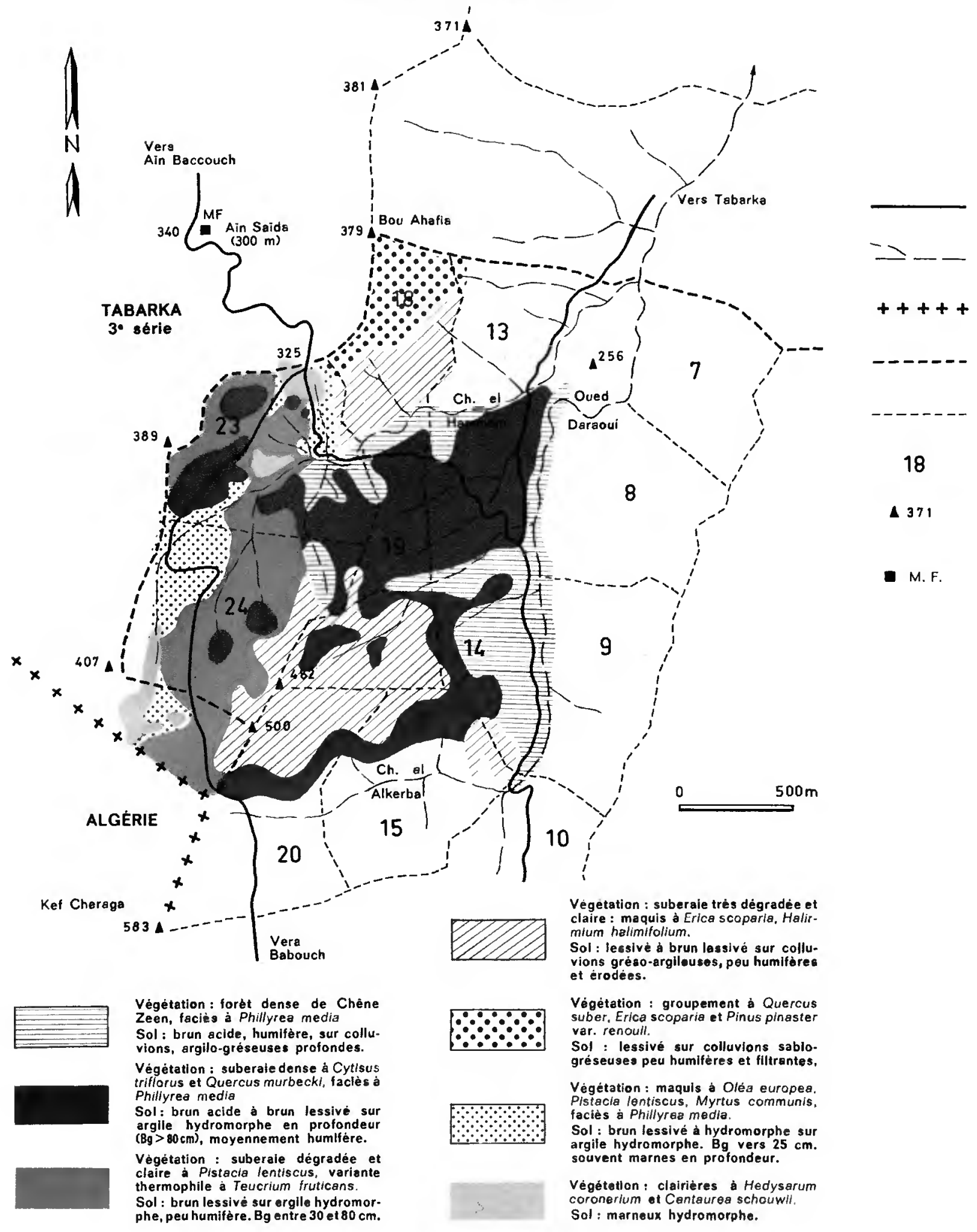




\section{CARTE D'UTILISATION}

Piste carrossable

Oued ou Chabet

Frontière algèro-tunisienne

Limite de subdivision forestière

Limite de parcelle

Numéro de parcelle

Sommet remarquable Cote d'altitude

Maison forestière
1
$N$
$N$ Vere

$$
\text { Ain Baccouch }
$$

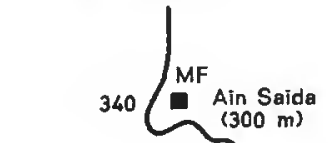

TABARKA

3* série

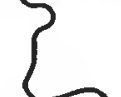

$x$

$\times$

407

$x$

$x$

1

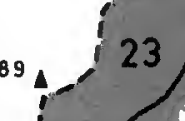

$-725$

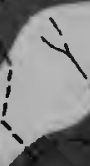

Bou Ahafia

3714

1 
ment dégradé par un peuplement artificiel, c'est-à-dire le reboisement en résineux ou en Eucalyptus.

A la suite des premières et multiples études de milieux qui démontrèrent l'hétérogénéité des stations et en particulier des sols, il est maintenant admis que transformation n'est pas obligatoirement synonyme de reboisement ; la destination finale de ces zones étant fonction des résultats des études de milieux qui devront y être entreprises.

En fait il s'agit d'un véritable micro-aménagement de ces quartiers de transformation (ou de ces zones hors-aménagement) puisque les études de milieux aboutissent à distinguer : utiliser,

- les zones réellement susceptibles d'être reboisées, ainsi que les essences à y

- les zones à vocation pastorale ou fourragère,

- les zones à mettre en défense et où la couverture végétale doit être maintenue en vue d'une protection contre l'érosion (active ou potentielle),

- les zones où l'on trouve des bouquets de peuplements ligneux en bon état, suffisamment vastes et denses pour pouvoir former ultérieurement des unités de gestion forestière,

- enfin les zones faisant l'objet d'une occupation humaine ou agricole illicite.

Ainsi, en même temps qu'elles sont la condition indispensable à la réussite immédiate des reboisements, les études de milieux, parce qu'elles permettent de séparer sur des critères scientifiques, des sols à vocation réellement forestière de ceux à vocation fourragère ou nulle, fournissent les éléments nécessaires aux aménagements pastoraux et au choix des emplacements des villages forestiers.

\section{TECHNIQUE D'ETUDE DES MILIEUX}

La technique d'étude des milieux que nous avons contribué à mettre au point à l'Institut de Reboisement de Tunis, est fondée sur la distinction et la délimitation d'unités homogènes de végétation dont on *étalonne " ensuite l'aptitude au reboisement par l'étude de quelques profils pédologiques. L'utilisation de photographies aériennes (2) permet la cartographie rapide de ces unités.

Tout en conservant une rigueur scientifique satisfaisante, cette méthode a été conçue pour être facilement utilisée par des agents de terrain peu spécalisés, du moins au départ, et pour ne mettre en jeu qu'un minimum de matériel. La distinction et la description des unités de végétation relèvent de techniques phytosociologiques simplifiées. Elles font appel simultanément à :

- des critères physionomiques de formations végétales,

— des critères plus purement phytosociologiques d'espèces caractéristiques et indicatrices,

- des critères écologiques simples, principalement topographiques et édaphiques.

On parlera ainsi par exemple d'un type de forêt ripicole dense à Quercus murbeckii et Prunus avium sur sol brun acide et humifère développé sur colluvium de pente triasique

(2) Photographies en noir at blanc au $1 / 25.000$ eu au $1 / 12.500$ e de l'Institut géographique national. 
ou, par exemple encore, d'un type de groupement de terres de culture sur sol marneux vertique à Hedysarum coronarium, Centaurea schouwii et Cynara cardunculus (3).

Sans entrer dans le détail de cette méthode, nous dirons qu'elle peut se résumer en trois phases principales :

- au bureau, étude des photographies aériennes et réalisation d'une carte topographique simplifiée où sont reportés les principaux éléments de la planimétrie, du parcellaire forestier et les contours des unités de végétation distinguées ;

- sur le terrain, repérage des unités distinguées sur la photo, inventaire et description de celles-ci, étalonnage de l'aptitude de chaque milieu par l'étude de quelques profils pédologiques ;

- au bureau, synthèse des travaux ; établissement de la liste des milieux, de leurs aptitudes et de leur vocation finale. Etablissement de la carte des milieux et de la carte d'utilisation.

Alors que la carte des milieux est un document sclentifique relativement détaillé, donnant pour chaque milieu distinct, sa description, son écologie sommaire et la valeur intrinsèque de son sol, la carte d'utilisation, qui servira pour toute la suite des travaux de reboisement, est beaucoup plus simple. Elle est particulièrement indispensable à la délimitation effective sur le terrain des zones à défricher, phase essentielle des opérations de reboisement.

Sur la carte d'utilisation, tous les milieux ayant des aptitudes identiques y sont regroupés. Les unités de trop faible superficie y sont négligées pour des impératifs économiques. Cette carte n'a de signification que dans le contexte économique actuel et compte tenu des essences de reboisement actuellement utilisées. De plus elle fait un choix entre plusieurs utilisations possibles.

Par planimétrie, on aura une idée approximative des surfaces des différents secteurs réellement plantables, ce qui est indispensable pour la prévision de la masse des travaux à effectuer, pour prévoir leur financement et pour l'estimation du nombre de plants de pépinière nécessaire.

Cette étude des milieux permet en outre de se rendre compte des possibilités existantes d'accès dans les différents secteurs qui seront réboisés et donc de prévoir les travaux d'infrastructure qui seront nécessaires (création de pistes nouvelles, raccordement au réseau existant, implantation éventuelle de parcellaire, etc...). Cette infrastructure à créer sera indiquée comme telle sur la carte d'utilisation.

\section{PLACE des etudes de MILIEUX dANS la suite des OPERATIONS de REBOISE- MENT}

La méthode élaborée par l'Institut de Reboisement de Tunis repose sur la constatation qu'un bon déroulement de tous les travaux de reboisement suppose que les études écologiques aient lieu plusieurs années avant le reboisement proprement dit.

Cela implique une planification poussée des travaux à effectuer et la constitution d'une " réserve " de zones ayant fait l'objet de telles études, et donc prêtes à être reboisées. Une telle réserve constitue le volant indispensable permettant, malgré la fluctuation inévitable des possibilités financières, de ne reboiser que des secteurs préalablement reconnus aptes.

(3) La distinctlon des dilferents types de mllleux, des differents types de sols, de leur Interelatlon et de leur potentiallté, appliquée IGi sur une vaste échelle pour les besolns des chantlers de rebolsement, s'appule sur les résultats déjâ acquis en phytosoclologie, pédologle el phytopédologie, lant dans le cadre de l'Institut de Reboisement de Tunis, qu'au cours de travaux antérieurs. 
L'étude des milieux n'est qu'une des phases du reboisement. Elle doit intervenir préalablement à toute une série d'opérations obligatoires. Ces opérations étant destinées à mettre en terre un plan adéquat, au moment opportun, pour une reprise optimale, sur un sol jugé apte à le recevoir et préparé convenablement dans ce but.

Décrire cette chaîne d'opérations élémentaires n'est pas notre propos. Nous voulons seulement insister sur l'opération qui doit suivre immédiatement l'étude des milieux et qui en est le corollaire : la délimitation effective sur le terrain des zones destinées à être reboisées.

Sans entrer dans le détail de la technique mise au point à l'Institut de Reboisement de Tunis, nous dirons seulement qu'elle consiste à séparer, au moyen de layons sommaires ouverts à travers la végétation et de marques de peintures voyantes apposées sur les pierres ou sur les troncs d'arbres, les parties destinées à être reboisées de celles qui devront conserver leur couverture végétale naturelle.

C'est bien entendu la (ou les personnes) qui a effectué l'étude des milieux qui est la plus qualifiée pour diriger les équipes de délimitation. Elle est en effet la mieux à même de reconnaître les milieux qu'il s'agit de délimiter.

A chaque utilisation différente du terrain correspondra une couleur de peinture (celle de la carte d'utilisation). Cela permettra le moment voulu, au chef de chantier de reconnaître les zones qu'il convient de défricher et quelle espèce de plant y mettre.

A titre d'illustration, voici les couleurs qui ont été conventionnellement adoptées (couleurs voyantes et que l'on peut facilement se procurer dans le commerce) :

- rouge pour tout ce qui doit être conservé,

- jaune pour les zones à vocation pastorale ou fourragère,

- blanc pour le Pin maritime,

- bleu pour le Pin pignon,

- rose pour le Pin d'Alep,

- noir pour le Cyprès.

De plus, dans un but de protection contre l'érosion, il est prévu de conserver sytématiquement une bande de végétation naturelle le long des oueds, des talus de piste, des ruisseaux et autour des griffes d'érosion. La largeur de ces bandes doit être fonction des risques d'érosion.

Tout en luttant efficacement contre les dangers d'érosion, ce réseau de bandes de végétation naturelle constitue un cloisonnement des reboisements, donc un dispositif de lutte contre les incendies. II permet en outre une meilleure intégration des reboisements au paysage, ce qui doit permettre de mieux sauvegarder leur aspect esthétique et donc la potentialité touristique de cette région et aussi concourrir à y préserver les équilibres biologiques.

Evidemment, tout ceci implique beaucoup de soins, un grand nombre de journées de travail à l'ha. et donc beaucoup d'argent. Mais, en fin de compte, étant donné les économies réalisées en ne reboisant que les terrains productifs, le procédé s'avère des plus intéressants.

\section{CONCLUSION}

II est devenu banal de dire qu'à notre époque, dans les pays fortement industrialisés comme l'Europe, sous la pression des exigences du développement économique et social, l'aménagement du territoire et la préservation de la Nature sont devenus une nécessité vitale. Cet angoissant problème est même devenu politique. 
Dans les pays en voie de développement, ce problème ne se pose pas encore avec la même acuïté. Pour qu'ils puissent brûler les étapes et rattraper aussi rapidement que possible le niveau que d'autres pays ont mis des siècles à atteindre, il leur est indispensable de procéder à la mise en valeur totale et intégrée de leurs ressources naturelles.

Les multiples expériences réalisées de par le monde ont suffisamment montré que les critères écologiques étaient la base la plus rationnelle pour toutes les études d'aménagement, par leur respect de la diversité et de l'originalité des milieux naturels.

A l'occasion des programmes de reboisement établis par le gouvernement tunisien, cette vérité s'est imposée une nouvelle fois.

\section{REMERCIEMENTS}

Nous tenons à remercier M. J. MARION, Directeur du projet F.A.O. "Institut de Reboisement " qui nous a conflé le sain de réaliser ces études de milieux dans les périmètres de reboisement.

Notre reconnaissance va également à tous les membres de la Recherche forestiére et de l'Administration des foréts tunisiennes, qui nous onl apporlé leur concours.

Je tiens à remercier très spécialement MM. P. DIMANCHE el A. SCHOENENBERGER, experts de la F.A.O. qui m'ont initié à la connaissance des sols et de la végétation du Nord de la Tunisie.

\section{Jean TIMBAL}

Chargé de recherches au C.N.R.F.

14, rue Girardet 54 - NANCY

\section{BIBLIOGRAPHIE}

\footnotetext{
COSSON (E.). - Rapport de la mission botanique chargée en 1883 de l'exploration du Nord de la Tunisle. Paris, Imprimerie Nationale, 1885.

DEBAZAC (E.F.). - La végétation forestiẻre de la Kroumirie. Annales de l'Ecole nallonale des eaux et forêts, t. 16, fasc. II, 1959, pp. 1-133.

FERRE (Y. de ). - Pin maritime ou Pin mésogéen en Alrique du Nord. $78^{\mathrm{e}}$ congrès des saciétés savantes, 1953.

Carte phyta-écalogique de la Tunisie septentrionale. Echelle $1 / 200.000^{\mathrm{e}}$. Natice délaillée (feuille II : BizerteTunis ; feuille III : Tabarka-Sauk-el-Arba). Annales de I'Instltut nallonal de la recherche agronomlque de Tunlsle, val. 40, fasc. 1, 1967.
} 